\title{
Norois
}

Environnement, aménagement, société

$217 \mid 2010 / 4$

Interroger les processus de valorisation des espaces urbains

\section{La Manufacture d'Armes de Saint-Étienne : un conflit mémoriel}

The weapons factory of Saint-Étienne: a memorial conflict

\section{Thomas Zanetti}

\section{(2) OpenEdition}

\section{Journals}

Édition électronique

URL : https://journals.openedition.org/norois/3467

DOI : 10.4000 /norois.3467

ISBN : 978-2-7535-1565-9

ISSN : 1760-8546

\section{Éditeur}

Presses universitaires de Rennes

\section{Édition imprimée}

Date de publication : 15 décembre 2010

Pagination : 41-55

ISBN : 978-2-7535-1340-2

ISSN : 0029-182X

Référence électronique

Thomas Zanetti, «La Manufacture d'Armes de Saint-Étienne : un conflit mémoriel », Norois [En ligne], 217 | 2010/4, mis en ligne le 31 décembre 2012, consulté le 13 janvier 2022. URL : http:// journals.openedition.org/norois/3467 ; DOI : https://doi.org/10.4000/norois.3467 


\title{
La Manufacture d’Armes de SAInT-Étienne : un CONflit MÉmoriel
}

\author{
Thomas ZanetTI \\ EA « Habiter » 2076 - Institut d'Aménagement du Territoire, d'Environnement \\ et d'Urbanisme de l'université de Reims (IATEUR) \\ (Université Reims Champagne-Ardenne), \\ 57 bis, rue Pierre-Taittinger - 51100 Reims, France \\ tomazanetti@hotmail.com
}

\section{RÉSUMÉ}

La Manufacture d'Armes de Saint-Étienne, un des plus célèbres symboles du passé industriel de la ville, a été, entre juillet 2004 et juin 2005, au cour d'un vaste conflit mémoriel. Sa réhabilitation, à partir de 2004, en équipement culturel et économique, dans le cadre d'un projet urbain soumis à de multiples enjeux, a donné naissance à une vive polémique autour de la question de la patrimonialisation de l'ensemble bâti. À la fois porteur de ressources de légitimation pour l'action publique contemporaine et de références identitaires pour une partie de la population locale, le lieu hérité est devenu l'objet de luttes d'appropriation, de qualification et de valorisation spatiale entre les collectivités locales qui portaient le projet et plusieurs associations de défense du patrimoine. Le déroulement controversé de sa mise en patrimoine, en tant que processus construit socialement et politiquement, permet alors de définir le rapport de forces qui s'institue au sein de la société locale quant au devenir des espaces hérités de l'industrie. Il traduit enfin un mécanisme d'instrumentalisation mémorielle et conduit à l'avènement de mémoires orphelines de patrimoine.

MOTS CLÉ : industrie - mémoire - appropriation de l'espace - projet urbain - conflit

\section{ABSTRACT}

\section{The weapons factory of Saint-Étienne: a memorial conflict}

The Weapons factory of Saint-Étienne, one of the most famous symbols of the industrial past of the city, was, between July, 2004 and June, 2005, in the heart of a vast memorial conflict. Its rehabilitation, since 2004, in cultural and economic equipment, within the framework of an urban project subjected to multiple stakes, gave birth to a deep debate around the question of its heritage recognition. At the same time carrier of resources of legitimization for the contemporary public action and identical references for a part of the local population, the inherited place became the object of fights of appropriation, qualification and spatial valuation between the local authorities in charge of the project and several associations of defense of the heritage. The controversial progress of its stake in heritage, as process builds socially and politically, allows then to define the balance of power which is established within the local society as for the future of spaces inherited from the industry. It translates finally a mechanism of memorial manipulation and leads to the birth of orphan memoirs of heritage.

KEY WORDS : industry - memory - appropriation of the space - urban project-conflict 
Un espace fortement investi pour son potentiel symbolique véhicule de multiples représentations collectives dans les différentes composantes de la société locale. L'action publique urbaine, en se saisissant de la « réserve de sens » inscrite dans le lieu, se prononce alors sur l'héritage transmis et se transforme en une « action patrimoniale de fait, une action qui évalue (ou dévalue) la valeur patrimoniale de la trace " (Colson et Roux, 1994). Cet article vise à analyser le déroulement d'un processus de valorisation d'un espace anciennement dévolu à l'activité industrielle, à travers sa mise en patrimoine et son renouvellement architectural et urbain. En abordant l'exemple stéphanois, on peut montrer de façon significative comment les valeurs véhiculées par un espace précis - celui de la Manufacture d'Armes - évoluent dans le temps, et comment une action contemporaine - ici, la mise en patrimoine - au service d'un projet d'avenir peut en grande partie immobiliser ces dernières. Il permet ensuite de saisir le rôle d'acteurs variés (l'État, les collectivités locales, le secteur associatif, etc.) dans la fabrique des valeurs attribuées à un lieu précis, ainsi quà leur évolution, et donc de questionner « les interactions possibles entre traces industrielles, groupes sociaux et projets urbains » (Valognes, 2006). Enfin, il illustre l'existence de stratégies matérielles et idéelles d'appropriation des espaces mises en œuvre par certains groupes sociaux qui, dans ce cas précis, s'opposent et font ainsi apparaître des luttes, des conflits pour l'appropriation d'un espace fortement disputé. Si l'on suit le raisonnement proposé par P. Melé (2005), l'entrée par les conflits rend possible l'analyse de la construction sociale du patrimoine. Ainsi, les polémiques qui naissent « pour la reconnaissance de tel ou tel élément du patrimoine, peuvent être considérées comme une entrée privilégiée pour une géographie sociale préoccupée par l'analyse de la façon dont les groupes sociaux s'approprient directement ou symboliquement l'espace urbain » (Melé, 2005).

À travers l'analyse des discours produits par les acteurs impliqués dans les champs de la politique urbaine et du patrimoine, le cas stéphanois permet donc d'interroger les rapports de force et de pouvoir qui s'instaurent lorsque se redéfinissent les valeurs et les représentations symboliques d'un lieu hérité, lorsque se confrontent, selon les impératifs du présent et les enjeux d'un avenir incertain, la « remise » et la « reprise» (Colson et Roux, 1994). L'apparition de stratégies de marquage social de l'espace urbain donne ainsi à voir des « capacités inégales des différents groupes sociaux à laisser une trace et à s'approprier les espaces les plus prestigieux » (Veschambre, 2002) qui s'expriment dans des « formes de domination symbolique qui s'exercent en instrumentalisant le patrimoine » (Melé, 2005).

\section{La Manufacture d'Armes de Saint-Étienne : un territoire-enjeu de la mémoire}

Un rapide détour par l'histoire stéphanoise (Fournial, 1976; Merley, 1990) permet de souligner la relation d'interdépendance qui unit l'industrialisation de la capitale ligérienne et son développement urbain. Le premier essor démographique d'envergure de Saint-Étienne, au Xvi ${ }^{\mathrm{e}}$ siècle, entretient déjà un lien avec une vocation industrielle affirmée, dû notamment à l'expansion du secteur de l'armurerie. Ce dernier (au même titre que la passementerie, la métallurgie et la quincaillerie) assure le démarrage de l'économie locale et joue un rôle décisif dans la croissance d'une ville qui n'était jusque-là qu'une petite bourgade enclavée dans ses montagnes. Le paysage stéphanois va d'ailleurs très vite porter les marques de cette épopée industrielle, tout comme la structure socio-spatiale de l'espace urbain, si bien que la capitale du Forez est aujourd'hui perçue comme « l'archétype de la ville développée et aménagée entre 1850 et 1970 pour répondre aux besoins des grands établissements industriels » (Dormois, 2009). Ainsi, les différentes activités vont se spécialiser par quartiers, permettant à cette occasion l'extension de la ville au nord. Au XVIII ${ }^{\mathrm{e}}$ siècle, Saint-Étienne va acquérir un rayonnement plus important encore grâce aux progrès continus de l'armurerie ${ }^{1}$. En effet, en 1764, neuf manufacturiers se regroupent en Manufacture Royale déte-

1. Le changement de nom de Saint-Étienne dans les dernières années de la Révolution, rebaptisée «Armeville » pendant plus d'une année, atteste du caractère central de cette activité. 
nant le monopole de la fourniture des armes pour le Roi, les puissances étrangères, la compagnie des Indes et la traite des Noirs. Cette Manufacture est en fait un ensemble d'établissements souvent de très petite taille. Au siècle suivant, l'armurerie stéphanoise s'organise principalement à partir de la Manufacture, qui devient propriété de l'État à partir de 1838. Grâce à une modernisation des équipements, elle était la plus importante en France et alimentait le marché national, mais aussi les colonies. De plus, elle était réputée pour la beauté de certaines de ses fabrications, notamment les armes de luxe incrustées, véritables chefs-d'œuvre des graveurs stéphanois. C'est d'ailleurs à cette époque que s'intensifie la relation entre l'art et l'industrie, avec la création de l'École des arts industriels et du Musée d'art et d'industrie, préfigurant le concept de l'écomusée tel qu'il est apparu au $\mathrm{Xx}^{\mathrm{e}}$ siècle.

Durant le premier conflit mondial, le secteur des armes va connaître de nouveau une forte croissance, Saint-Étienne étant appelé à devenir l'arsenal de la France en guerre. Cet essor se répétera lors de la seconde guerre mondiale, mais ce pilier symbolique de l'économie stéphanoise va ensuite décliner, les effectifs baissant d'un quart entre 1962 et 1975. La situation économique à Saint-Étienne dans les années 1970 est caractéristique d'une crise d'une puissance sans précédent. La région vit alors une récession qui frappe les principaux secteurs industriels et amène à l'effondrement des bases traditionnelles de l'économie locale. Durant la décennie 1980, l'agglomération stéphanoise affiche le visage d'une vieille région industrielle très durement touchée par une crise économique qui traduit l'épuisement du modèle productif fordiste. Malgré un agenda politique local où la question de la reconversion économique devient rapidement prioritaire, le paysage urbain portera durablement les stigmates de son déclin, avec notamment la multiplication des friches industrielles.

Cette origine industrielle du développement urbain explique en grande partie la faible présence à Saint-Étienne de monuments issus du patrimoine «classique » et ancien. Les éléments urbains et architecturaux appartenant aujourd'hui au patrimoine stéphanois datent donc en majorité des XIX et $\mathrm{XX}^{\mathrm{e}}$ siècles, époque où la ville se développe avant tout grâce à son activité industrielle prospère. Pour orienter son extension selon un axe nord-sud, Saint-Étienne adopte en 1792 le plan en damier de Dalgabio, choix qui signe l'avènement de l'urbanité stéphanoise. Le plan Dalgabio crée ainsi la ville moderne et «met en place les formes urbaines et architecturales les plus caractéristiques de Saint-Étienne, celles qui allaient assurer son identité » (Bonilla et al., 2005). Il correspond aujourd'hui à l'élément le plus ancien du patrimoine urbain local. Les quartiers passementiers sont une autre spécificité architecturale et urbaine issue de l'activité artisanale et urbaine. Situés sur les pentes des collines entourant la cité, ils se singularisent par la construction d'immeubles-ateliers alignés qui disposent d'étages surélevés et de hautes fenêtres. D'autres quartiers du Saint-Étienne du XIX ${ }^{e}$ siècle sont liés à une activité industrielle particulière, lien traduit par l'homogénéité de leur paysage urbain. C'est d'ailleurs particulièrement le cas de ceux où sont implantés les armuriers.

Le paysage urbain a lui aussi gardé de nombreuses traces (crassiers, friches, usines...) de son riche passé industriel. D’abord considérées comme les stigmates du déclin de l'activité économique locale (Gay, 1996), elles ont progressivement été assimilées à des opportunités potentielles pour le développement futur de la ville. La ville a enfin hérité de son passé un fort patrimoine immatériel, même si ces éléments n'ont été que tardivement perçus comme des biens patrimoniaux réclamant une conservation. La classe ouvrière, malgré son caractère hétérogène et son éclatement en plusieurs corporations, a marqué Saint-Étienne de son empreinte, lui léguant un important héritage social (identité, culture, ensemble de valeurs...) et technique (savoir-faire, esprit d'innovation...). Ce rapide inventaire du patrimoine stéphanois souligne la continuité du lien que celui-ci cultive avec l'activité industrielle, principale source de l'essor économique, démographique et urbain durant les $\mathrm{XIX}^{\mathrm{e}}$ et $\mathrm{XX}^{\mathrm{e}}$ siècles. Suite à l'élargissement de la notion patrimoniale, à « la triple extension typologique, chronologique et géographique des biens patrimoniaux » (Choay, 1992), et à la consécration de monuments appartenant à un passé sans cesse plus proche du présent, la ville a lentement découvert la richesse d'un patrimoine aujourd'hui appréhendé comme une ressource économique et symbolique, «à la fois héritée et stratégique »(Greffe, 2000). Saint-Étienne s'est 
ainsi vu attribuer le label «Ville d'Art et d'Histoire » en décembre 2000, pour son riche patrimoine du XIX ${ }^{\mathrm{e}}$ siècle.

L'histoire de la Manufacture d'Armes de Saint-Étienne (MAS) reste quant à elle assez méconnue de nos jours, ou tout du moins fragmentée, ce qui s'explique notamment par son statut militaire, couvert par le secret-défense et de ce fait fermé au public, ainsi que par l'éparpillement de ses archives. Il y a donc très peu d'écrits qui retracent le passé de la MAS, et il n'y a pas encore d'histoire « officielle ${ }^{2}$ » de la manufacture, ni d'ouvrage faisant référence en la matière. Par exemple, on ne sait pas exactement quelles sont les évolutions architecturales successives qui ont modifié l'ensemble industriel et les données concernant les effectifs de "manuchards » - surnom donné aux employés de la Manufacture - sont également assez variables. On peut toutefois dessiner de manière assez précise les contours du passé de la MAS, à travers les grandes dates de son histoire. À partir de 1864, se construit à Saint-Étienne, sur un terrain de 12 hectares, une usine dont le but est de regrouper l'ensemble des ateliers réunis sous le titre de manufacture royale d'armes, alors encore disséminés dans différents quartiers de la ville. Les différentes composantes de l'ensemble, achevé en 1870, font apparaître une association de bâtiments aux fonctions différenciées : lieux de productions (usines, ateliers), lieux de vie et d'habitation (cantine, dortoirs, réfectoire), bâtiments réservés à l'administration. La réalisation de la nouvelle manufacture d'armes met en place l'utilisation des principes d'organisation rationalisée du travail, avec notamment le contrôle du travail et de la production. Pour ce qui est des effectifs, plusieurs chiffres nous renseignent sur l'importance de cet ensemble industriel : 10000 ouvriers en 1890, 16000 durant la première guerre mondiale, 10000 en 1940. C'est seulement après 1945 que s'amorce le lent mais inéluctable déclin de cette industrie. La deuxième partie du $\mathrm{Xx}^{\mathrm{e}}$ siècle verra la manufacture nationale poursuivre avec un bonheur inégal la fabrication des armes légères suivant les époques de guerre, de paix ou d'occupation. L'établissement fermera en 1990, dans un contexte de crise de l'industrie de l'armement, consécutive à la chute du mur de Berlin et l'effondrement du bloc communiste. En 2000, l'EDIACAT (service d'archives militaires et imprimerie pour les publications) s'implante sur le site dans le but de sauvegarder des emplois, qui sont aujourd'hui au nombre de 147 et dont la majorité sont d'anciens « manuchards ». La trajectoire suivie par la Manufacture est donc représentative de celle d'une économie locale suscitée à l'origine par l'État et faisant la promotion d'une forte intégration des PMI « dans des systèmes hiérarchiques et verticaux dominés par une ou plusieurs grandes firmes qui s'inscrivaient dans la logique des champions nationaux » (Le Galès, 2006). L'effondrement des bassins traditionnels d'activité dans les années 1980 a entraîné une désindustrialisation brutale du territoire et a causé la déstructuration complète du tissu économique stéphanois, composé d'un grand nombre de sous-traitants agissant pour d'importants donneurs d'ordres. Enfin, la crise locale du fordisme a provoqué la présence d'un «système de relations sociopolitiques marqué par l'évitement et le cloisonnement [...] peu propice à l'élaboration de stratégies collectives » (Béal et al., à paraître). Cette organisation particulière des rapports politiques, sociaux et économiques a éloigné la possibilité d'émergence d'un acteur collectif et a constitué un frein à la construction d'une stratégie concertée quant au devenir de ce tissu soudainement plongé dans le déclin.

La relative méconnaissance de l'histoire du site a permis une grande latitude aux groupes sociaux qui se sont attelés à une construction de son passé. C'est le cas pour des faits historiques précis mais aussi pour les significations du plan d'ensemble, et particulièrement en ce qui concerne la dimension symbolique des maisons de direction. Ainsi, les associations de défense du patrimoine voient dans l'ensemble architectural une véritable cité industrielle dans l'esprit du courant de pensée utopiste, en tout cas l'expression, sous le Second Empire, de la cité industrielle idéale, la MAS pouvant même correspondre selon eux à la réalisation de l'utopie de Claude-Nicolas Ledoux. La morphologie urbaine est un autre point important dans la réflexion menée par ces « entrepreneurs de mémoire locale » (Saez et Glevarec, 2002). Pour ces derniers, la Manufacture d'Armes

2. Nous devons toutefois signaler une publication récente (sur laquelle nous reviendrons), éditée par le Musée d'Art et d'Industrie de Saint-Étienne, et dont l'ambition est de donner un éclairage sur le passé du site de la MAS. 
de Saint-Étienne doit une grande partie de son originalité à un plan d'ensemble de facture classique et de conception géométrique, construit tel un échiquier géant intégrant les espaces pleins et les espaces vides. Enfin, les bâtiments abritant les maisons des directeurs, placés à l'entrée du site, sont perçus comme les symboles de la hiérarchie socio-spatiale. Selon les associations, la présence de ces pavillons est un élément fondamental pour la qualité de l'ensemble, car elle lui confère sa cohésion. L'analyse historique menée par le tissu associatif révèle une attention particulière portée aux dimensions architecturales et urbaines du site, et dans une moindre mesure, à sa dimension sociale, ce qui n'est pas le cas pour tous les acteurs qui se sont mobilisés dans une relecture de l'histoire de l'espace industriel. En effet, si l'on suit par exemple le discours de la conservatrice du Musée d'Art et d'Industrie de Saint-Étienne, c'est une autre partie de l'usine qui doit retenir l'attention, celle qui regroupe les lieux de production. Ces derniers auraient vu la mise en place d'une organisation du travail poussée qui aboutira au taylorisme. Cette consécration de l'intérêt historique des bâtiments de production renvoie quant à elle à une volonté de sauvegarde de la mémoire technique stéphanoise. Ce choix de l'héritage productif comme trace à valoriser

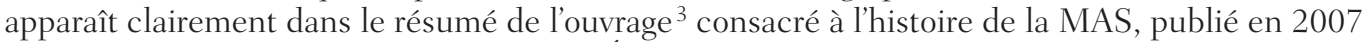
par le musée d'Art et d'Industrie de Saint-Étienne. Le site industriel y est en effet décrit par les termes suivants : " usine alors à la pointe de la modernité », "production mécanisée à échelle industrielle », « culture technologique novatrice », " mise en perspective historique du design des armes de guerre, né de la confrontation à la mécanisation »... Enfin, divers acteurs locaux (des représentants associatifs, notamment) ont voulu mettre en avant la mémoire sociale du site. Pour ces derniers, l'ensemble de la population de la ville est attaché à la MAS, site qui rappelle mieux que tout autre l'illustre passé industriel de la cité. La persistance d'éléments symbolisant l'histoire ouvrière stéphanoise et sa tradition de solidarité, tel le monument aux morts situé sur l'esplanade qui signale l'entrée du site, est considérée comme une opportunité pour inscrire durablement ces valeurs dans l'identité locale.

Cette diversité de sélections mémorielles révèle l'existence d'une pluralité d'acteurs issus de la sphère patrimoniale locale et engagés à divers degrés de responsabilité dans la réflexion sur le devenir du site. Ces différents protagonistes se sont saisis du passé des lieux et en ont proposé des visions parfois divergentes. Ainsi, la communauté d'agglomération a remis en cause une partie du travail historique réalisé par les associations, notamment sur les évolutions successives qu'a connu le site. Ces tentatives de subjectivation de l'histoire de la MAS apparaissent comme des mises en perspective historiques qui font ressortir des mémoires pouvant se contredire. Il y a donc une tension entre différentes interprétations du passé de l'ensemble industriel qui indiquent une volonté de répondre à d'autres enjeux que la simple connaissance de l'histoire.

\section{Un espace investi par un projet d'avenir : de la « ville noire » à la Cité du Design}

La Cité du Design est au cœur du projet «Saint-Étienne Métropole Design ». Projet urbain et architectural de grande envergure, elle constitue, selon la communauté d'agglomération de Saint-Étienne, « l'élément fédérateur des ambitions culturelles et économiques de l'agglomération, qui souhaite être identifiée comme la métropole française du design ${ }^{4}$ ». Si l'on en croit la communication établie par la Ville de Saint-Étienne, c'est dans la capitale ligérienne qu'est né le design. Les pouvoirs publics locaux ont ainsi pu la proclamer à maintes reprises « berceau du design industriel ${ }^{5} »$. Pour justifier ces affirmations, la municipalité a effectué, depuis le début des

\footnotetext{
3. La Manufacture d'Armes de Saint-Étienne. La révolution des machines (1850-1870). Coédition Un, Deux... Quatre Éditions et Musée d'Art et d'Industrie de Saint-Étienne, 184 p. 2007.

4. On peut retrouver l'expression de ce vœu dans divers documents, publiés au cours de l'année 2004-2005, ayant trait à la politique de l'instance intercommunale, et dans les plaquettes de présentation du projet de la Cité.

5. Affirmation qui traverse également divers documents de communication institutionnelle.
} 
années 2000, une plongée dans l'histoire de sa ville. Ainsi, différents corps de métiers (passementiers, armuriers, fabricants de cycles) particulièrement présents dans l'histoire économique de la région ont été qualifiés de pionniers du design industriel. Celui-ci ferait donc partie de l'histoire de Saint-Étienne qui a toujours favorisé un dialogue entre l'art et l'industrie, et ce projet d'avenir ne sortirait donc pas de nulle part, mais serait le continuum d'une tradition séculaire de savoir-faire, de qualification, d'esprit de créativité et d'inventivité.

Cette histoire locale du design est légitimée par l'existence de deux lieux symboliques : le Musée d'Art et d'Industrie (MAI) et la Manufacture d'Armes de Saint-Étienne (MAS), présentés comme des endroits où s'est ébauchée la notion moderne de design. Le premier, créé au milieu du XIX ${ }^{e}$ siècle, est considéré comme un lieu où s'est construite la notion du design telle qu'on la connaît aujourd'hui. En favorisant la rencontre entre productions artistiques et techniques, en conjuguant le souci du beau et la recherche de l'utilité à des fins d'efficacité économique et industrielle, la MAI aurait mis en place des initiatives qui « annoncent déjà le Bauhaus, l'esthétique et donc, le design », comme on peut le lire dans un document publié par la communauté d'agglomération.

La Manufacture d'Armes de Saint-Étienne (MAS) entretiendrait également des liens avec cette activité. L'industrie d'armement y aurait engagé une recherche de l'esthétique, des formes nouvelles mais aussi une organisation plus poussée de la production. Ce lien entre rationalisation de la production industrielle et considération plus importante pour l'aspect visuel, déjà exprimé par l'action du MAI, a permis de faire de la MAS un haut-lieu du passé « design » de Saint-Étienne. Signalons également l'école des beaux-arts, autre lieu-ressource de cette continuité historique autour de l'industrie du design. Celle-ci fut créée dans un esprit de coopération entre industriels et artistes, ces derniers étant amenés à réfléchir sur la conception de produits issus de l'industrie. Enfin, la biennale du Design de Saint-Étienne, dont la première édition date de 1998, est le dernier événement qui doit instituer la ville comme capitale française du design.

Avec son projet d'avenir axé sur le design, initié depuis le début des années 2000, c'est fort logiquement que Saint-Étienne s'appuie sur son histoire industrielle, sur les savoir-faire traditionnels de ses ouvriers et artisans, et sur l'appréhension d'une continuité historique entre le design moderne et les particularismes des activités stéphanoises anciennes (passementerie, armurerie, etc.). Le passé constitue ainsi une ressource pour un présent en quête d'images locales spécifiques et « la posture face à l'héritage devient celle d'une exploitation de la différence sur fond de continuité »(Colson et Roux, 1994). La mémoire technique est réinvestie selon les impératifs du présent et l'incertitude liée à l'avenir, devenant ainsi une mémoire nourricière pour le projet de développement de l'agglomération. La Manufacture, elle, se voit investie a posteriori d'un ensemble de valeurs de contemporanéité (Riegl, 1984). La grande majorité des acteurs impliqués dans la connaissance du passé local ne remettent pas en cause cette continuité entre savoir-faire traditionnels et positionnement actuel sur l'activité design, mais certains dénoncent une campagne de communication opportuniste mise en œuvre par les collectivités locales.

La Cité du Design, équipement phare du projet de développement de l'agglomération, sera confrontée à un ensemble d'enjeux, dont la réussite est nécessaire pour permettre à l'agglomération stéphanoise d'être identifiée comme «Ville Design ». Pour Saint-Étienne Métropole, principale institution en charge du projet, ce dernier doit d'abord relever un défi économique, avec l'objectif de devenir un acteur majeur de la mutation de l'ensemble de la région grâce au renouvellement du tissu productif et de la base industrielle. La Cité se fixe ainsi comme ambition de développer, auprès des acteurs économiques et sociaux du territoire, l'utilisation du design dans le cadre d'une démarche innovante, en tant que plus-value apportée à la production. Ensuite, la Cité vise à amener une valeur ajoutée aux structures d'enseignement et de recherche, en suscitant la création d'une offre de formation supérieure de très haute qualité dans le champ du design. Elle propose également, en lien avec l'École Supérieure d'Art et de Design de Saint-Étienne, ses propres enseignements et parcours pédagogiques. Le projet d'agglomération « design », qui s'apparente à un véritable projet de société, a en outre pour objectif de diffuser sur l'ensemble du territoire 
stéphanois une culture design. En tant que symbole de ce projet, il incombe ainsi à la Cité du Design de proposer des actions en faveur du grand public, afin de répondre à des enjeux en termes de tourisme urbain. Cet équipement de grande envergure doit donc, pour accroître ses chances de succès, être approprié par l'ensemble de la population de l'agglomération stéphanoise, faire sens pour les habitants. Enfin, la Cité du design correspond à une volonté farouche de changer l'image de la ville pour qu'elle soit désormais caractérisée par sa postmodernité, objectif qui a pu autrefois être assigné à la politique sportive de l'agglomération (Merle, 2009). Elle ambitionne de rayonner sur la scène internationale, ou du moins aspire à y être visible. Ce rayonnement à grande échelle, après avoir donné lieu à un travail sur son passé, passe par un changement en profondeur de l'identité de l'agglomération. C'est donc un vaste projet de marketing urbain qui induit des préoccupations de labellisation résultant d'une fabrique locale de normes approuvées à l'échelle internationale (Devisme et al., 2007). Ces pratiques signalent le crédit accordé au marketing identitaire territorial dans la définition du contenu des projets urbains actuels qui se fixent comme but de concilier exemplarité rassurante d'expériences étrangères réussies ${ }^{6}$ et singularité du contexte local comme vecteur de différenciation et de visibilité. Comme la souligne N. Arab (2007), il s'agit ici de parvenir à un équilibre entre emprunt et dépassement. Les édiles locaux entendent conférer à leur représentation du futur stéphanois une matérialité monumentale et veulent ériger la Cité en symbole de cette vision moderniste, le projet design étant porteur d'une dimension attractive dans les relations que cette activité entretient avec l'esthétique, l'art et la modernité. L'aspect futuriste de l'enveloppe architecturale conçue manifeste ce désir de passage à une ville post-moderne, et autorise en outre de développer des actions de communication à grande échelle. Cette détermination à imposer une nouvelle identité correspond aussi à un objectif de mixité sociale "par le haut ", c'est-à-dire à une captation des classes moyennes, voire des très recherchées classes créatives (Florida, 2003).

Le projet design serait donc guidé par un besoin de reconnaissance « post-moderniste », et constituerait un vecteur de promotion pour l'agglomération dans l'optique d'attirer de nouveaux habitants, séduits par cette nouvelle «Ville Design ». À la Cité s'adjoint en effet la réalisation d'un programme de logements de standing, laissant présager de la gentrification du quartier de la Manufacture et, à terme, le départ des classes les plus populaires vers des zones urbaines périphériques. Le contenu du projet implique donc d'« accepter que le renouvellement urbain soit synonyme de renouvellement social » (Bonneville, 2004).

Le projet architectural de la Cité du Design (photo 1), réalisé par l'agence LIN, consiste en la construction d'une platine aux dimensions imposantes ${ }^{7}$ et s'implante dans la cour d'honneur de la Manufacture d'Armes de Saint-Étienne, dont on a vu plus haut les caractéristiques principales, notamment en terme de considérations patrimoniales. Le marquage de l'espace par la modernité architecturale doit donner à voir la monumentale matérialisation du projet d'avenir, la Cité étant le vecteur le plus visible de la diffusion d'une culture Design. Ce projet architectural est donc une projection matérielle de la nouvelle identité de l'agglomération, empreinte de modernité. En tant que principale représentation du changement d'image de la ville et de la continuité historique de l'activité design sur le même lieu, il aspire à l'iconicité. En quelque sorte, les pouvoirs publics, au premier rang desquels on trouve la communauté d'agglomération, font le pari que la Cité du Design représentera un jour le symbole du patrimoine stéphanois du $\mathrm{XxI}^{\mathrm{e}}$ siècle, puisque cette réalisation doit être le témoignage d'une culture collective appropriée par l'ensemble de l'agglomération.

L'espace d'implantation de la Cité du Design est donc emblématique de la mutation urbaine de la ville, il est amené à constituer une vitrine de son renouvellement urbain et démographique, dont la visibilité conditionnera en partie l'amélioration de l’image de Saint-Étienne, encore considérée

6. Dans le cas de Saint-Étienne, on pense évidemment à la référence de Bilbao, les élus locaux se plaisant aussi à citer Lille ou Glasgow comme modèle.

7. $36 \mathrm{~m}$ de large, $220 \mathrm{~m}$ de long, et 5 à $6 \mathrm{~m}$ de hauteur, pour une surface totale de $12000 \mathrm{~m}^{2}$ 


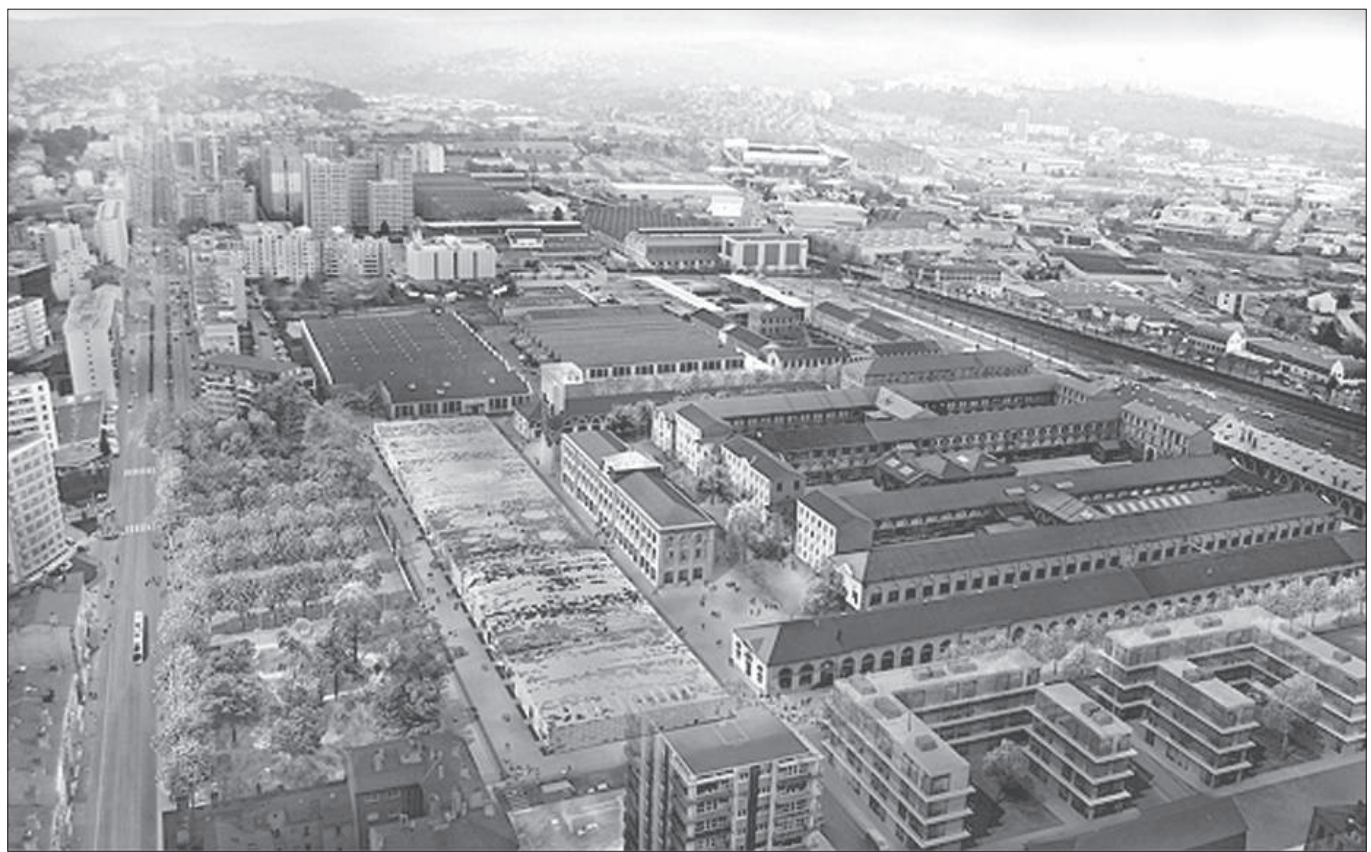

Photo 1 : Vue aérienne prospective de la Cité du Design et de la Manufacture Forward-looking aerial view of the city of the design and the weapons factory

comme une «ville noire » (Vant, 1981). Le pari que s'est donc vu proposer le lauréat du concours est celui de l'instauration d'un dialogue architectural entre les bâtiments de la manufacture présents sur le site et ceux de la future Cité du Design, dans le cadre d'une «symbiose imposée où il est escompté que l'intérêt suscité par l'œuvre du présent se répercute sur l'œuvre ancienne, annonçant ainsi une dialectique »(Choay, 1992). La priorité reste toutefois la composition d'un ouvrage représentatif de la nouvelle modernité stéphanoise, étendard du retournement d’image d'une ville qui ambitionne de devenir une des capitales mondiales du design. En s'installant sur le site de la MAS (fig. 1), ce projet soulève la question de la relation entre passé et modernité; entre le patrimoine, sa conservation, et la création architecturale contemporaine dans un contexte de renouvellement urbain. Le projet architectural de la Cité du design permet ainsi d'aborder l'enjeu mémoriel inhérent à toute opération d'aménagement, et renseigne plus largement sur le rapport qu’entretient la société stéphanoise, à travers ses élus, vis-à-vis de son passé.

\section{Processus de patrimonialisation et conflit mémoriel}

À travers l'implantation de la Cité du Design, la Manufacture d'Armes de Saint-Étienne s'est retrouvée au cœur d'une controverse qui s'est cristallisé autour de la question du patrimoine. Les associations et, à un moindre degré, les habitants de la ville, se sont opposés à l'implantation de la Cité du Design, celle-ci devant entraîner la destruction des maisons de direction et des bâtiments administratifs.

Malgré une année de combat contre ce projet architectural, les collectivités locales en charge de sa réalisation sont "passées à l'acte » et ont démoli les quatre bâtiments en catimini, le matin du 22 juin 2005 (photo 2). Un retour sur la nature conflictuelle de ce processus de catégorisation et d'appropriation spatiale, sur le déroulement de la mise en patrimoine de la Manufacture, ses 
Figure 1 : La Manufacture d'Armes, espace d'implantation de la Cité du Design

The Weapons factory, the space of setting-up of the City of the Design

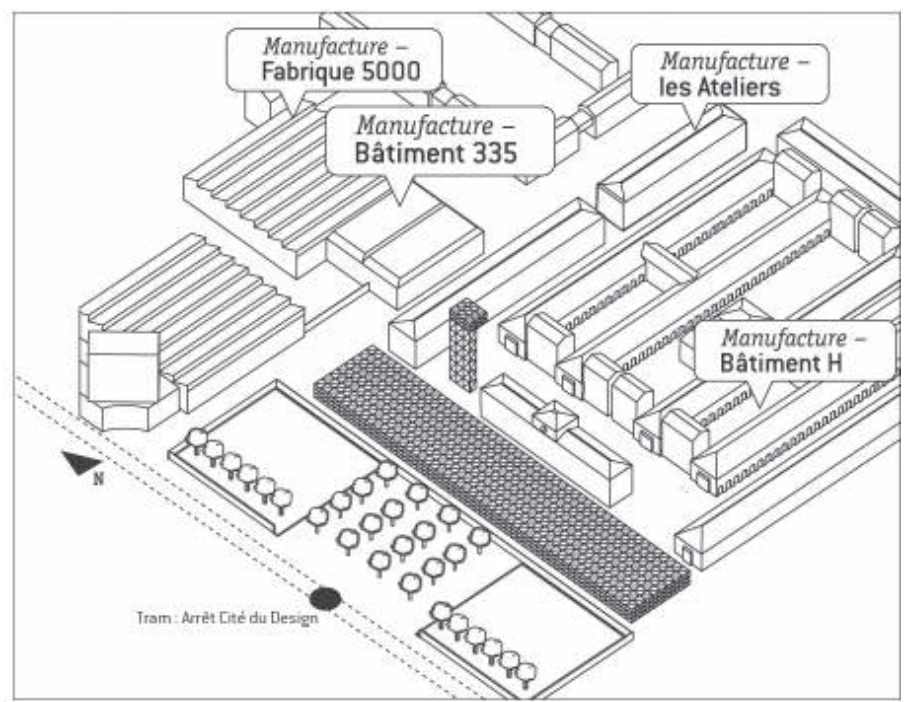

conséquences et ses significations, permet de saisir l'espace comme enjeu de pouvoir, d'interroger la construction locale de rapports socio-spatiaux hiérarchiques, et de déterminer les motivations qui président au contrôle spatial.

Il existe à Saint-Étienne une multitude d'acteurs investissant le champ du patrimoine, regroupés au sein de ce que l'on pourrait nommer une nébuleuse patrimoniale. Le premier acteur identifié est l'État et ses services déconcentrés qui interviennent dans le cadre de missions clairement définies : conseil, contrôle et conservation du patrimoine. Toutefois l'acteur central du champ patrimonial reste la municipalité stéphanoise, même si elle ne détient pas de compétence en matière conservatoire. C'est en effet l'échelon communal qui établit la politique de mise en valeur dans les domaines de la culture et du patrimoine, et dont la mise en œuvre est assurée par des adjoints municipaux. La collectivité dispose également d'autres structures intervenant sur ces questions. C'est le cas de l'unité Ville d'Art et d'Histoire (VAH), chargée de mettre en place la convention du label du même nom, obtenu pour la présence à Saint-Étienne d'un important patrimoine issu

Photo 2 : Un bâtiment administratif détruit le 22 juin 2005

An administration building destroyed on June 22nd, 2005

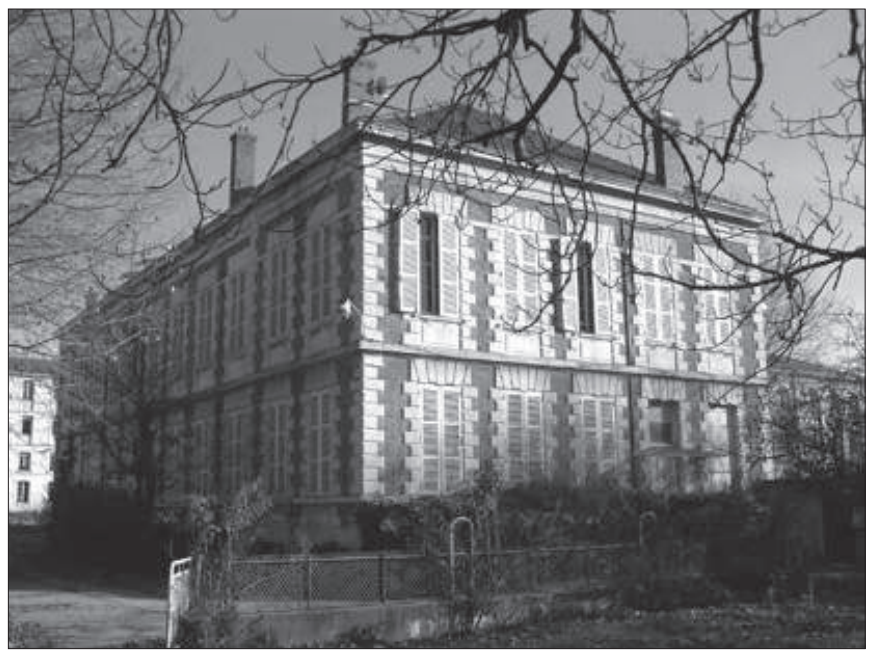


des $\mathrm{XIX}^{\mathrm{e}}$ et $\mathrm{XX}^{\mathrm{e}}$ siècles; du Musée d'Art et d'Industrie, qui présente des collections liées à l'industrie stéphanoise ; du Musée de la Mine, qui se donne pour but de préserver l'identité minière du bassin de la Loire; et enfin des Archives municipales. Le tissu associatif prend également part à la nébuleuse locale du patrimoine. On peut ainsi dégager deux associations principales : les Amis du Vieux Saint-Étienne (AVSE), association créée en 1930, a pour objet « d'étudier, de protéger, de conserver et de faire connaître l'histoire et le patrimoine de la région stéphanoise ${ }^{8} »$. Depuis 1988, l'Association de Recherche, de Concertation et d'Ouverture (ARCO) a quant à elle pour rôle la valorisation de l'image extérieure de Saint-Étienne. Ces structures réalisent généralement un gros travail d'érudition sur divers thèmes, participent également à une démarche de médiation et assument un rôle de leadership dans la représentation du patrimoine et de la mémoire collective. L'Université, par le biais de différents laboratoires de recherche ${ }^{9}$ travaillant sur la thématique patrimoniale, a également permis une meilleure connaissance de l'histoire locale. C'est même le milieu universitaire qui a été le lieu de " découverte » du patrimoine stéphanois, en favorisant par son action une première prise de conscience patrimoniale à l'échelon politique. Enfin, la communauté d'agglomération, Saint-Étienne Métropole, est le dernier acteur qui agit de manière ponctuelle dans la politique patrimoniale locale, même si elle n'en détient pas la compétence, essentiellement à l'occasion de la mise en œuvre de grands projets urbains, comme celui de la Cité du Design ${ }^{10}$.

Les entretiens réalisés auprès d'acteurs locaux du patrimoine font apparaître une multitude d'approches du patrimoine local. La quasi-totalité des représentants des diverses structures locales citent le patrimoine industriel, et évoquent son caractère récent, mais tous n'accordent pas la même importance à ses dimensions matérielles et immatérielles. De même, les composantes architecturales et urbaines, paysagères, sociales et identitaires ne sont pas toujours définies dans des termes semblables, et n'occupent pas des positions équivalentes dans la hiérarchie du patrimoine local. On observe par conséquent des conceptions diverses du passé, une variété de ses représentations, qui révèlent une différenciation dans les rapports à l'histoire et à la mémoire. La montée des préoccupations patrimoniales à Saint-Étienne ces dernières années a contribué à l'éclosion d'une véritable " arène patrimoniale » (Roth, 2003). Cette arène ou cet "écosystème » (Greffe, 2000) est traversé par un champ de forces qui crée des tensions, des conflits autour des questions patrimoniales, elles-mêmes liées aux processus de revalorisation qui touchent à des lieux hérités du passé.

Comme nous l'avons vu dans une première partie, il existe un enchevêtrement d'histoires et de mémoires inscrit dans l'espace de la Manufacture. Seul un processus de patrimonialisation, en tant que mécanisme social et politique de construction mémorielle, pouvait arbitrer entre les différentes interprétations et consacrer ainsi une vision spécifique de l'histoire. Une démarche de classement a abouti à l'inscription partielle au titre des Monuments Historiques de la MAS. Le périmètre de protection concerne la totalité de l'ensemble industriel à l'exception des maisons de direction et des pavillons administratifs, c'est-à-dire les bâtiments dont la destruction était prévue pour permettre l'implantation de la future Cité du Design. Cette décision pose la question des conditions de l'élection patrimoniale. Le choix de faire disparaître des éléments qui paraissaient réclamer une consécration patrimoniale au moyen d'un régime de protection, a pour première conséquence d'anéantir la cohérence générale de l'ensemble architectural. La symbolique du lieu et son marquage social ont ainsi été effacés, étant donné que c’est la partie noble de la manufacture, celle qui représente le pouvoir et la mise en scène de la hiérarchie des espaces, qui a été exclue de la consécration patrimoniale.

Cette mise en patrimoine partielle s'apparente ensuite à une mise à mal de la mémoire ouvrière et sociale d'un site emblématique de l'histoire industrielle stéphanoise. On observe en effet un

8. Site Internet de l'association.

9. Et notamment l'Institut des Etudes Régionales et du Patrimoine (IERP), dont la directrice siégeait à la Commission Régionale du Patrimoine et des Sites (CRPS) durant le conflit de la Manufacture.

10. Ou de l'achèvement de l'église Saint-Pierre du Corbusier à Firminy, autre élément mobilisé dans le cadre de la «stratégie design » de l'agglomération. 
attachement des salariés de la Manufacture à leur ancien lieu de travail et une persistance de la mémoire ouvrière dans un espace industriel qui, du fait de sa situation géographique, de la réputation de ses productions, de la longévité de son histoire et du nombre important de stéphanois qui y ont travaillé, occupe une place prépondérante dans l'histoire ouvrière de la ville. L'ampleur de la réaction citoyenne - non prise en compte par les élus - suite à l'annonce des démolitions (lettres au maire, 8000 signatures à la pétition contre les destructions...) est venue prouver cet attachement populaire à la Manufacture. La MAS serait donc un lieu capital dans l'histoire industrielle stéphanoise et il existerait un lien affectif envers le passé ouvrier de la ville, d'autant plus que beaucoup de lieux industriels ont déjà disparu. Ici ressurgit la question de la relation que la société stéphanoise entretient avec son passé, et de la teneur de la dialectique contradictoire entre nécessité de la mémoire et volonté de l'oubli. Le contexte de tension dans lequel s'est inscrit la patrimonialisation de la Manufacture peut amener à la conclusion suivante : la mémoire institutionnelle du territoire n'arrive pas encore à faire émerger positivement tous les épisodes de crises et de ruptures qui jalonnent une histoire récente encore douloureuse (Ricœur, 2000), car évoquant à la fois les réminiscences d'une activité industrielle disparue, les références classiquement associées au monde du travail (luttes ouvrières, etc.) et les souvenirs véhiculés par une mémoire jugée embarrassante, à savoir celle des conflits armés. Si l'historicisation peut « émaner d'un traumatisme collectif, d'un événement séparateur qui pousse à renouer le fil du temps en s'appuyant de façon privilégiée sur les traces matérielles et mémorielles » (Fabre, 2001), elle n'a pu dans le cas de la manufacture faire l'économie d'une instrumentalisation qui révèle quant à elle une stratégie d'effacement (Debray, 1999). L'euphémisation des pans les plus difficiles de l'histoire industrielle locale renvoie à une tentative des élus d'exorciser ce passé embarrassant. Le travail de deuil (Ricœur, 2000) engagé par la société stéphanoise resterait en ce sens inabouti et correspondrait à la manifestation d'une « forme de thérapie par l'oubli qui sélectionne certains évènements pour en cacher d'autres » (Rautenberg, 2003), cette dernière s'exprimant dans un processus de patrimonialisation « destiné à sceller l'oubli de ce qui divise » (Tisseron, 1999).

La patrimonialisation de la MAS apparaît donc comme un processus mêlant des intentions idéologiques et un pragmatisme économique, orchestré selon des impératifs d’image de marque et de distinction territoriale, et qui ne doit en aucun cas contraindre la capacité du site industriel à devenir une ressource pour le présent et pour l'avenir. Car la mémoire du site, du moins celle qui a été promue au rang de patrimoine, à savoir la mémoire technique, constitue un enjeu de légitimation de l'action publique et une garantie pour la réussite du développement de l'ensemble de l'agglomération. La mémoire, enjeu fondamental du renouvellement urbain, revêt ici une complexité particulière du fait de l'existence de deux constructions symboliques (MAS et Cité du design) amenées à cohabiter sur un même espace. L'échec du dialogue entre les éléments qui matérialisent ces différentes mémoires a conduit à une situation d'enchevêtrement mémoriel concurrentiel.

La première rupture qui a entraîné ce conflit a eu lieu bien avant la présentation du projet lauréat en Juillet 2004, lors de la phase d'écriture du cahier des charges. Ce dernier stipulait la présence obligatoire de la future Cité à un endroit précis du site, à savoir la cour d'honneur, sans que des mesures conservatoires ne soient imposées. La faible prise en compte de la dimension patrimoniale du site d'implantation de la Cité dans le processus de programmation et de conception du projet s'explique en partie par la connaissance limitée du site et l'absence de consultation des experts locaux du patrimoine lors de la rédaction du cahier des charges. La seconde rupture provient de la composition du jury retenu pour désigner le lauréat du concours d'architecture de la Cité du Design. En effet, aucun spécialiste de l'histoire stéphanoise n'en faisait partie, ce qui n'a pas joué en faveur des architectes ayant présenté un projet plus attentif aux traces matérielles et mémorielles présentes sur le site. Suite à l'annonce du lauréat du concours, les associations de défense du patrimoine ont engagé un combat dans le but d'éviter les atteintes portées à la Manufacture, utilisant de nombreux moyens pour arriver à leurs fins. Elles ont tout d'abord produit une alternative au projet retenu, consistant en la proposition d'une autre implantation permettant la 
sauvegarde des bâtiments menacés. Les associations ont ensuite entrepris d'informer la population stéphanoise des dangers qu'encourait la Manufacture, dans l'objectif de susciter une réaction populaire.

La première initiative dans ce sens a été la production d'une pétition, intitulée «Touche pas à notre Manu ». Lancée juste après l'annonce du projet lauréat, elle a recueilli 8000 signatures en une année et a été suivie d'un référendum par sondage, dans le but de connaître l'opinion des Stéphanois. Puis, les associations ont écrit des lettres aux différentes instances responsables du projet (sénateurmaire de Saint-Étienne, direction de Saint-Étienne Métropole, président du Conseil Régional...), expliquant les raisons de leur opposition et demandant de ne pas procéder aux démolitions. Elles ont ensuite réalisé un important travail d'érudition visant à établir une meilleure connaissance du passé du site industriel et à démontrer la grande valeur historique du patrimoine existant, recherche qui a profité de l'expertise de plusieurs spécialistes locaux, issus notamment du milieu universitaire. Enfin, le dernier moyen d'opposition a été la demande de classement en urgence du site, sur laquelle nous reviendrons ensuite. Les associations stéphanoises, irritées d'avoir été mises à l'écart de la phase de conception de la Cité, ont donc initié un débat polémique par la production d'un contrediscours patrimonial, et ont ainsi provoqué un conflit mémoriel en utilisant tous les recours, toutes les « armes » existantes (juridiques, démocratiques, intellectuelles...) pour faire évoluer le projet lauréat vers un plus grand respect de l'histoire du site et convertir ses responsables à la " religion patrimoniale »(Choay, 1992). On peut voir dans ce combat une résistance à l'imposition d'une histoire institutionnelle à visée hégémonique, mais également un processus d'acquisition de pouvoir et de reconnaissance dans la sphère patrimoniale et mémorielle locale.

Un retour sur le déroulement du classement à l'Inventaire Supplémentaire des Monuments Historiques (IMSH) fait apparaître la mise en tension concurrentielle, le conflit entre les différentes mémoires matérialisées par les éléments physiques de la MAS. Dès 1997, les Amis du Vieux Saint-Étienne (AVSE) demandent à la DRAC une protection sur les bâtiments de façade et sur les jardins de ce site jugé emblématique de la ville. Cette requête n'aboutit pas pour plusieurs motifs : persistance sur le site d'une activité, complexité du statut foncier de la Manufacture qui appartient au Ministère de la Défense... Suite à l'annonce du projet lauréat et des démolitions que ce dernier entraîne, les associations vont engager une nouvelle démarche de classement en urgence de l'ensemble du site industriel. Elles vont ainsi écrire au préfet de la région Rhône-Alpes pour demander la saisine de la Commission Régionale du Patrimoine et des Sites (CRPS) afin que cette dernière se prononce sur l'éventualité d'une protection de la Manufacture et l'inscrive à son ordre du jour. Après plusieurs ajournements, le dossier de la Manufacture d'Armes de Saint-Étienne va finalement être soumis à la CRPS en Juin 2005. Cette dernière, qui n'a pas de pouvoir décisionnel mais dont l'avis consultatif est généralement toujours suivi, est composée d'élus, de fonctionnaires du ministère de la Culture, d'experts scientifiques et de représentants d'associations patrimoniales (ici au nombre de trois). Elle est présidée par le Préfet de région qui arrête l'inscription. La décision de la commission va revêtir une importance capitale, en tant que sommet du rapport de forces établi autour de la question de la protection de la MAS et en tant qu'issue législative du conflit patrimonial. La CRPS va se prononcer, à une courte majorité, en faveur de l'inscription de l'ensemble du site de la manufacture, à l'exception des deux bâtiments administratifs et des deux maisons de directeurs, c'est-à-dire ceux dont la démolition était prévue. La cour d'honneur, lieu d'implantation de la Cité du Design, est elle aussi exclue de la mesure qui consacre la mise en patrimoine. La délibération de la commission s'apparente donc à un classement fait en fonction du projet, et l'on peut assimiler cette décision à la volonté des élus locaux d’imposer une sélection mémorielle spécifique.

La destruction des quatre bâtiments, le matin du 22 juin 2005, va marquer définitivement ${ }^{11}$ l'issue d'un conflit patrimonial qui aura duré une année. L'historique de la démarche de classement

11. Même si l'on peut faire la supposition que le conflit a survécu à la destruction des quatre bâtiments, et que ses conséquences ont perduré, notamment dans la défaite électorale du maire de Saint-Étienne aux municipales de 2008. 
aura prouvé la volonté politique d'imposer une sélection mémorielle spécifique et sa supériorité dans le déroulement du conflit. La délibération de la CRPS a engendré la suprématie de l'interprétation de l'histoire stéphanoise portée par les élus, la patrimonialisation signant l'officialisation du «travail de mise en histoire des lieux pris en charge par les institutions »(Bensa, 2001). Le pouvoir politique constituerait alors le groupe porteur de mémoire le plus puissant et, en s'octroyant le droit de décider quels souvenirs doivent être retenus, il s'apparenterait en quelque sorte au titulaire d'une compétence mémoire. En arbitrant le conflit mémoriel à l'œuvre sur un site simultanément chargé de sens dans les consciences collectives et de vertus symbolisant la projection du territoire dans l'avenir, le pouvoir politique démontre son contrôle de la redéfinition du passé dans le présent et s'affirme comme seul juge de la "rentabilité du temps qui passe » (Carballo et Emelianoff, 2002). Grâce à l'outil de patrimonialisation, il évalue la valeur des biens hérités et légitime des pratiques de domination socio-spatiales. En proclamant la souveraineté du contemporain, la politique patrimoniale met le passé à distance et inscrit l'espace dans un choix identitaire, choix qui fonde sa temporalité.

Depuis une décennie, Saint-Étienne, dans un double mouvement, s'est d'une part attaché à se construire une nouvelle image, fondée sur la modernité, et s'est d'autre part lentement découvert une richesse patrimoniale, dont la composante industrielle pouvait même être identifiée comme une ressource capable de participer au renouvellement du positionnement identitaire local. Aujourd'hui, dans un contexte de compétitivité territoriale accrue et de course exacerbée à la réussite métropolitaine, le désir de changement radical des édiles stéphanois serait toujours présent, mais s'appuie paradoxalement sur une prise en compte sélective de l'héritage local, et fait ainsi apparaître un double besoin contradictoire de gommer et d'invoquer son passé.

Saisie par l'action publique urbaine contemporaine, la Manufacture d'Armes de Saint-Étienne est devenue un espace à fort enjeu et à forte cohabitation mémorielle où l'histoire a été revisitée par différents acteurs. La mise en tension de mémoires portées par les associations et d'autres produites par la municipalité a conduit à un processus de mise en patrimoine qui, en tant que mécanisme social et politique de construction mémorielle, a eu pour conséquence l'officialisation d'un passé se pliant aux exigences du présent (Bensa, 2001). La patrimonialisation partielle d'un espace symbolisant des mémoires différenciées apparaît alors comme une manière de se positionner vis-à-vis de l'héritage transmis par la présence sur un lieu de traces matérielles issues du passé. Le projet d'avenir opérerait alors une sélection de l'héritage à transmettre, en l'inscrivant dans une continuité historique jugée valorisante. La patrimonialisation, en tant que ressource pour qualifier et valoriser un espace, permet donc l'objectivation d'une production mémorielle subjective. Par sa partialité et sa sélectivité, ce mécanisme de qualification traduit un contexte d'appropriation de l'espace qui donne naissance à des luttes de reconnaissance mises en œuvre par les différents groupes sociaux, et crée in fine des mémoires orphelines de patrimoine.

La consécration de la mémoire technique, portée par la puissance aménageuse, traduit alors la position prééminente de la sphère politique locale dans la construction d'un ensemble de valeurs et de représentations symboliques dont l'espace est ensuite investi. À Saint-Étienne, le caractère éminemment politique de cette fabrique du passé met à jour un « enjeu de contrôle de la re-présentation du passé par le présent»(Bonniol, 2001). Cette domination d'une représentation spécifique du passé, produite et imposée par les collectivités publiques, a donné lieu à l'émergence au sein du groupe social ouvrier, représenté par le tissu associatif local, d'un sentiment de privation et de mise à distance qui s'explique par la mise à mal de la mémoire ouvrière du site. À travers cette confiscation de la mémoire ouvrière par les élites locales, on retrouve l'idée d'une manipulation politique de l'histoire et de la culture (Hobsbawm et Ranger, 2006), mais également celle de la « crise de visibilité » (Verret, 1996) d'une classe ouvrière stéphanoise pourtant encore importante numériquement. La résistance opérée par les associations a quant à elle donné naissance à un conflit patrimonial et mémoriel illustrant les oppositions existantes au sein de la société locale, oppositions qui s'avivent lors des processus de qualification et de valorisation d'espaces hérités, de lieux porteurs de souvenirs émanant d’un passé successivement honni et glorifié, ainsi 
que d'espoirs pour l'avenir. La présence de stratégies conflictuelles d'appropriation sociale dans la production de l'action collective patrimoniale manifeste enfin l'absence d'un régime urbain (Elkin, 1987; Molotch et Logan, 1987; Stone, 1989) stable présidant à la valorisation des espaces industriels. Ce défaut de concertation et de coalition dans le champ du renouvellement urbain éloigne la possibilité de voir s'édifier une gouvernance patrimoniale stéphanoise (Zanetti, 2008), même si l'électrochoc suscité par le sacrifice de la Manufacture pourrait conduire à faire évoluer la fabrique patrimoniale locale.

\section{Bibliographie}

Arab N., 2007. À quoi sert l'expérience des autres? Bonnes pratiques et innovation dans l'aménagement urbain, Espaces et Sociétés, nº 131, p. 33-47.

Beal V., Dormois R., Pinson G., à paraitre. Redeveloping Saint-Étienne. The weight of the inherited structure of social and political relationships in a French industrial city, dans Burkner H.-J. (dir.), Urban trajectories under conditions of decline. Economic crises and demographic change as trigger for new concepts of regeneration, Leipzig, Coll. Conditio Urbana, LIT-Verlag.

Bensa A., 2001. Fièvre d'histoire dans la France contemporaine, dans Bensa A., Fabre D. (dir.), Une histoire à soi : Figurations du passé et localités, Paris, Ed. MSH, p. 1-12.

Bonneville M., 2004. Les ambiguïtés du renouvellement urbain en France, Annales de la recherche urbaine, n 97 , p. 7-16.

Bonilla M., Tomas F., Vallat D., 2005. Cartes et plans. Saint-Étienne du XVIII siècle à nos jours. 200 ans de représentation d'une ville industrielle, Saint-Étienne, Presses Universitaires de Saint-Étienne, 182 p.

Bonniol J.-L.., 2001. La fabrique du passé. Le Larzac entre mémoire, histoire et patrimoine, dans Bensa A., Fabre D. (dir.), Une histoire à soi : Figurations du passé et localités, Paris, MSH, p. 169-198.

Carballo C., Emeliannof C., 2002. La liquidation du patrimoine ou la rentabilité du temps qui passe, Annales de la recherche urbaine, $\mathrm{n}^{\circ}$ 92, p. 49-57.

Chony F., 1992. L'allégorie du patrimoine, Paris, Seuil, 272 p.

Colson D., Roux J., 1994. Héritier d'une tradition industrielle: Un enjeu temporel pour la cité. Le cas du Marais à Saint-Étienne (Loire), Lille, Presses universitaires, 287 p.

Debray R., 1999. L'abus monumental, Paris, Fayard, 439 p.

Devisme L., Dumont M., Roy E., 2007. Le jeu des bonnes pratiques dans les opérations urbaines entre normes et fabrique locale, Espaces et Sociétés, n 131, p. 15-31.

Dormois R., 2009. Les coalitions dans l'analyse des politiques urbaines post-keynésiennes, Métropoles, $\mathrm{n}^{\circ} 4$, $26 \mathrm{p}$.

Elkin S., 1987. City and regime in the American republic, Chicago, University of Chicago Press, 227 p.

Fabre D., 2001. L'histoire a changé de lieux, dans Bensa A., Fabre D. (dir.), Une histoire à soi : Figurations du passé et localités, Paris, Ed. MSH, p. 13-44.

Florida R., 2003, The Rise of the Creative Class: And How It's Transforming Work, Leisure, Community and Everyday Life, Basic Books, 434 p.

Fournial E., 1976. Saint-Étienne : histoire de la ville et de ses habitants, Roanne, Ed. Horvath, 427 p.

GAY G., 1996. La ville industrielle, de l'exception à la banalisation: la fin des grandes usines métallurgiques de l'Ondaine et de Saint-Chamond, Revue de Géographie de Lyon, vol. 71, n³ 3, p. 197-207.

Greffe X., 2000. Le patrimoine comme ressource pour la ville, Annales de la recherche urbaine, ${ }^{\circ}$ 86, p. 29-38.

Hobsbawn E., Ranger T., 2006. L'invention de la tradition, Paris, Ed. Amsterdam, 370 p.

Le Gales P., 2006. La restructuration des PMI à Saint-Étienne après la crise : traces du passé et limites de l'intégration horizontale, Sociologie du travail, vol. 48, n 1, p. 17-36.

Melé P., 2005. Conflits patrimoniaux et régulations urbaines, La Lettre ESO, n² 23, Université de Tours, p. $51-57$. 
Merle S., 2009. Politiques d'équipements sportifs et évolution post-industrielle en région stéphanoise, Métropoles, $\mathrm{n}^{\circ}$ 4, $18 \mathrm{p}$.

Merley J., 1990. Histoire de Saint-Étienne, Toulouse, Privat, 318 p.

Molotch H. L., Logan J. R., 1987. Urban fortunes. The political economy of place, Berkeley, University of California Press, 383 p.

Rautenberg M., 2003. La rupture patrimoniale, Paris, À la Croisée, 173 p.

Ricceur P., 2000. La mémoire, l'histoire, l'oubli, Paris, Le Seuil, 675 p.

Riegl A., 1984. Le culte moderne des monuments, Paris, Le Seuil, 122 p.

Rотн C., 2003. Patrimonialisation et reconstruction d'un espace politique : les arènes patrimoniales. Communication au séminaire La patrimonialisation et après?, Université Lumière Lyon 2, p. 16-21.

Saez G., Glaverec H., 2002. Le patrimoine saisi par les associations, Paris, La Documentation française, $412 \mathrm{p}$.

Stone C., 1989. Regime politics: governing Atlanta 1946-1988, Lawrence, University Press of Kansas, 314 p.

Tisseron S., 1999. Comment l'esprit vient aux objets, Paris, Aubier, 231 p.

Valognes S., 2006. De la Société Métallurgique de Normandie à Effiscience, Annales de la recherche urbaine, $n^{\circ} 101$, p. 66-74.

VANT, 1981. Imagerie et urbanisme: recherche sur l'exemple stéphanois, Saint-Étienne, IERP, 656 p.

Verret M., 1996. La culture ouvrière, Paris, L'Harmattan, 296 p.

Veschambre V., 2002. Une mémoire urbaine socialement sélective, Annales de la recherche urbaine, $\mathrm{n}^{\circ}$ 92, p. 65-73.

ZANETTi T., 2008. La gouvernance patrimoniale stéphanoise : le consensus au prix du conflit?, Culture and local gouvernance, vol.1, n², p. 139-151.

Cet article a été reçu le 15 avril 2010 et définitivement accepté le 13 décembre 2010. 